\title{
Temperature-dependence of polymorphism in electrospun nanofibres of PA6 and PA6/clay nanocomposite
}

\author{
Gyeong-Man Kim ${ }^{a^{*}}$, G. H. Michler ${ }^{a}$, F. Ania ${ }^{b}$, F. J. Balta Calleja ${ }^{b}$ \\ ${ }^{a}$ Department of Physics, Martin-Luther-University Halle-Wittenberg, \\ D-06099 Halle/S., Germany \\ ${ }^{\mathrm{b}}$ Instituto de Estructura de la Materia, CSIC, c/ Serrano 119, \\ E-28006, Madrid, Spain
}

* Corresponding author. Address: Department of Physics, Martin-Luther-University Halle-Wittenberg, D-06099 Halle/S., Germany

Tel.: +493461 462571; fax: +493461462535

E-mail: gyeong.kim@physik.uni-halle.de (g.-M. Kim). 


\section{Abstract}

Polymorphism found in nanofibres of polyamide 6 (PA6) and PA6/clay nanocomposite (PA6-NC), prepared by an electrospinning process, was studied by transmission electron microscopy (TEM) and variable-temperature wide angle X-ray scattering (WAXS), and compared with the polymorphic changes occurring in the preelectrospun bulk materials. TEM results, concerning morphology and dispersion of the nanoclays, reveal that the produced electrospun nanofibres have an average diameter of $50 \mathrm{~nm}$ and the dispersion of nanoclays is much better achieved in the electrospun PA6-NC fibres than in the pristine PA6-NC. According to WAXS measurements, both types of electrospun nanofibres predominantly consist of $\gamma$-form crystals of PA6. Upon heating, from room temperature to the melting point, a number of successive transitions are observed for both systems, namely: crystalline $\gamma$ to $\alpha^{\prime}$, $\alpha^{\prime}$ to $\alpha$ and $\alpha$ to the "amorphous" $\delta$-form due to breakage of hydrogen bonds. On subsequent cooling, it has been observed, for the first time, that the development of crystalline forms for both systems is quite different from each other. The molten electrospun pure PA6 fibres firstly crystallize in the high temperature $\alpha^{\prime}$-form and then they show the room temperature $\alpha$-form. For these nanofibres, during a temperature cycle of heating and cooling, the initial $\gamma$-form crystals completely turn into the $\alpha$-form crystals as in bulk PA6. In contrast, for the electrospun nanofibres of the PA6-NC, the $\gamma$-form crystals are preserved after completing a thermal cycle down to room temperature. The present findings on the evolution of polymorphism in the electrospun nanofibres of both systems provide useful information regarding their use as reinforcing elements in polymer composites.

Keywords: Electrospinning; Nanocomposites; Nanofibers; Polymorphism 


\section{Introduction}

Polyamide 6 (PA6), due to its excellent physical properties, e.g., high fatigue strength, low coefficient of friction, enhanced toughness (depending on degree of crystallinity), and high resistance to a wide spectrum of fuels, oils, and chemicals, is a commercially important semicrystalline polymer [1]. The production of polymernanofiller composites, the so-called polymer nanocomposites (PNCs), is a relatively new line of research and most promising to improve the mechanical properties of PA6 [2-4]. Nanofillers, in general, are defined as exhibiting at least one dimension in the range of $1-100 \mathrm{~nm}$, which is the case of nanoclays (layered silicates) [5, 6]. Only small amounts of nanofillers (typically $1-4$ vol.\%), due to their nanometre size, high specific surface area and the associated predominance of interfaces, are capable of significantly affecting the structure and morphology of PNCs at a molecular scale [7, 8]. This means that they can influence material properties at levels which cannot be reached by traditional micron-size fillers. The resulting PNCs exhibit an excellent properties profile relevant to a wide diversity of industrial applications; i.e., high stiffness, chemical and thermal resistance, dimensional stability, reduced water absorption, as well as improved electric and optical properties.

The role of nanofillers for structural applications is not only limited to increasing the polymer matrix stiffness, but also to enhancing toughness driven by new energy dissipation mechanisms [7, 9-11]. Therefore, the development of PNCs, with a good balance of properties, demands to a high degree the coupling of processing with morphological and micromechanical control. For a rational design of new composites, at least two essential morphological requirements should be met: a) a uniform dispersion of nanofillers to avoid large agglomerates and high stress concentrations, and b) good interfacial bonding between the fillers and the polymer matrix to achieve effective load transfer across the filler-matrix interface [12-14]. Unfortunately, nanofillers are often dispersed in the polymer matrix in the form of agglomerates. This strong tendency to agglomerate reduces significantly their ability to bond with the matrix due to less contact area and therefore decreasing the effective aspect ratio of the reinforcement. Moreover, under an external load the stresses will be readily concentrated around such agglomerates, which in turn generally leads to a premature failure of the system. 
In order to suppress the tendency towards nanofiller agglomeration, electrospinning (ES) is being used as an alternative technique [15-20]. Up to now it is well established that the ES process provides not only a homogeneous dispersion within the fibres of nanofillers, such as layered silicates [21-23], carbon nanotubes [24-26] and many others [27], but also yields a drastic decrease in the fibre diameter down to several tens of $\mathrm{nm}$. The resulting nanofibres are continuous (and thus of extremely high aspect ratio) and with a large specific surface area (and thus strongly interacting with the environment). Since the early work of Griffith on the strength of fibres depending on fibre diameter [28], it is well known that such strength exponentially increases with decreasing fibre diameter; this is due to the fact that the probability of occurrence of a critical defect (and thus of failure) decreases rapidly with decreasing size for a given defect concentration. More recently, $\mathrm{Li}$ at al. have also found an increase in Young's modulus with decreasing fibre diameter in electrospun nanofibres of PA6 and PA6-NC [21]. Furthermore, based on our previous work [22] on the deformation behaviour of poly(methyl methacrylate) (PMMA)/Namontmorillonite (Na-MMT) nanocomposite electrospun fibres, and by observing an "in situ" stretched single fibre under transmission electron microscopy (TEM), it has been shown that while the bulk nanocomposite generally deforms in a brittle manner, the nanocomposite electrospun fibres deform by shear yielding in the form of necking. This unique energy absorbing mechanism ends up with an improvement of the toughness of the nanofibres. Based on the foregoing, the nanofibres containing nanofillers produced by ES can be considered as one of the most promising reinforcing elements for composite materials.

It is well known that polymorphism is one of the factors which determines the deformability of semicrystalline polymers [29]. Typically, PA6 exhibits polymorphic structures which are attributed to strong intermolecular interactions, such as hydrogen bonding between neighbouring amide groups. The monoclinic a-form preferentially develops at high crystallization temperatures or low cooling rates from the molten state. At low crystallization temperatures or rather high cooling rates, PA6 crystallizes in the pseudo-hexagonal $\gamma$-form. In the $\gamma$-form crystals, non-planar polyamide molecules adopt a parallel-chain arrangement of hydrogen bonding, whereas the polyamide molecules in the monoclinic a-form are planar, all-trans and packed in the more stable antiparallel chain arrangement of hydrogen bonds. The $\alpha$ - 
form is more stable than the $\gamma$-form, presumably because of the shorter and stronger hydrogen bonds [30] and the lower energy of the fully extended all-trans conformation of its chains. It is well known that the impact strength of a PA6 sample, containing mostly $\gamma$-form crystals, is markedly higher than that of PA6 with $\alpha$-form crystals, because the interchain interactions in the $\gamma$-form are smaller than those in the $\alpha$-form.

Various studies, by Li at al.[21] and Fong at al. [23], on the crystalline structure of the electrospun nanofibres of PA6 [31] and PA6-NC modified with MMTs, have demonstrated that $\gamma$-phase crystallites are most likely to appear in both systems due to the ultra-large draw ratio of electrospinning and the rapid solvent removal during formation of fibres. Before the electrospun nanofibres are used as reinforcing elements in composites, it is important to investigate the influence of temperature on polymorphism. As far as we know, there are still very few reports on this subject [32].

The aims of the present study are the following: 1) to prepare electrospun nanofibres of PA6 and the PA6/clay nanocomposite; 2) to investigate the polymorphism of PA6NC by means of wide angle X-ray scattering (WAXS); 3 ) to examine by TEM the dispersion of nanoclays in PA6-NC, as well as in its electrospun nanofibres; 4) to study the influence of temperature on the crystalline structure of the electrospun nanofibres of PA6 and PA6-NC by real time WAXS; and 5) to compare the results obtained in the electrospun nanofibres with those found in their bulk materials prior to electrospinning.

\section{Experimental Section}

\subsection{Materials}

PA6 $\left(M_{\mathrm{n}}=29300, \mathrm{MFI}=1.2\right)$ was a commercial grade from Honeywell. The nanoclay product used, obtained from Southern Clay Products Inc., was an organically modified montmorillonite (MMT) prepared by a cation exchange reaction between sodium montmorillonite and bis(hydroxyethyl)-(methyl)-rapeseed quaternary ammonium chloride (cation exchange capacity $=95$ mequiv. $/ 100 \mathrm{~g}$ clay, organic content $=34.6 \mathrm{wt} \%)$. The polymer nanocomposite (PA6-NC) containing $3.2 \mathrm{wt} \%$ organoclay was prepared by melt-blending using a Haak, co-rotating, intermeshing twin-screw extruder. Compounding was carried out at a barrel temperature of $240{ }^{\circ} \mathrm{C}$, 
a screw speed of $280 \mathrm{rpm}$, and a feed rate of $980 \mathrm{~g} / \mathrm{h}$. The extruded composite pellets were injection moulded to get the standard tensile specimens using an Arburg Allround 305-210-700 injection moulding machine. The details of the nanocomposite processing are described elsewhere $[33,34]$.

\subsection{Electrospinning process}

To obtain electrospinnable solutions, pure PA6 and its PA6-NCs with $3.2 \mathrm{wt} \%$ nanoclays were dissolved in formic acid to prepare $10 \mathrm{wt} \%$ solutions. These solutions were vigorously stirred with a magnetic stir bar for at least $24 \mathrm{~h}$ at room temperature, followed by sonication for $30 \mathrm{~min}$ to ensure homogeneity. It must be noted that sonication can produce chain scission, in particular for the high molecular weight fractions. In our case, however, this possibility did not seem to hinder the capability of obtaining electrospun fibers. Electrospinning was carried out at room temperature in a vertical spinning configuration, using a $1 \mathrm{~mm}$ inner diameter flat-end needle with a $5 \mathrm{~cm}$ working distance. The applied voltages were in the range from 3 to $20 \mathrm{kV}$, driven by a high voltage power supply (Knürr-Heizinger PNC, Germany). The electrospun fibres were collected either directly on Cu-grids or on aluminium foil.

\subsection{Transmission electron microscopy (TEM)}

The samples for the bulk PA6/NC were cryogenically microtomed at a temperature of $-80^{\circ} \mathrm{C}$ into ultra thin sections of about $50 \mathrm{~nm}$ thick using a diamond knife in a Leica Ultracut E Microtome. Sections were collected on the copper TEM grids and then examined by TEM (JEOL 2010) with an acceleration voltage of $200 \mathrm{kV}$. In order to characterize the dispersion of nanoclays within the electrospun fibres, the pure PA6 and PA6-NC fibres were directly electrospun on Cu-grids and then investigated by TEM without any chemical treatment. The diameter of the electrospun fibres and their distribution were analyzed by measuring over 200 fibers from randomly recorded TEM micrographs using an image analysis software (Analysis, Soft Imaging System Co. Germany).

\subsection{Wide angle $X$-ray scattering (WAXS)}

Wide-angle X-ray scattering was performed with a Seifert XRD 3000 using CuKa radiation ( $\lambda=1.54056 \AA)$. The tube source was operated at $40 \mathrm{kV}$ and $30 \mathrm{~mA}$. Scans were run in the low angle region of $2 \theta<10^{\circ}$ to study the nanofiller dispersion, and in 
the high angle region $10^{\circ}<2 \theta<40^{\circ}$ to follow the crystal form changes in the presence of nanoclays. A step size of $0.05^{\circ}$ and a dwell time of 1 second were used. The study of the temperature variation of the crystalline structure of the electrospun nanofibres, with and without nanoclays, was carried out by WAXS using a synchrotron radiation source in a double focusing mirror monochromator camera of the soft condensed matter beam line A2 (HASYLAB) at the DORIS III storage ring (DESY, Hamburg). A monochromatic radiation with a wavelength of $0.15 \mathrm{~nm}(8 \mathrm{keV})$ was selected. The beam size at the samples surface was $2 \times 1 \mathrm{~mm}^{2}$. Scattering patterns along the equator were recorded using a linear position sensitive detector, corrected for fluctuations in intensity of the primary beam and background. X-ray accumulation time for each frame was set to 30 seconds, with a waiting time between two consecutive frames of 30 seconds. From room temperature up to $230^{\circ} \mathrm{C}$, the heating rate was $3 \mathrm{~K} / \mathrm{min}$. On cooling, a much faster rate $(\sim 30 \mathrm{~K} / \mathrm{min})$ was used. Position calibration was performed using the standard crystalline diffraction peaks of $\mathrm{PET}$. The corresponding $d$-spacings were obtained from the scattering angle $2 \theta$ of the diffraction peaks according to Bragg's law: $d=\lambda /(2 \sin \theta)$.

\section{Results and discussion}

\subsection{Morphology of the bulk materials and their electrospun nanofibres}

The dispersion of nanoclays in an injection moulded PA6-NC is shown in Figure 1. It is clearly seen that the individual exfoliated nanoclays $(1 \mathrm{~nm}$ thick black lines in the TEM micrograph), together with two and three layer stacks (intercalated nanoclays), are homogeneously well-dispersed in the polymer matrix. The micrograph reveals a typical mixed nanomorphology, in which numerous exfoliated nanoclays and fewer portions of intercalated ones coexist. The insert in Figure 1 illustrates a WAXS pattern for the bulk PA6-NC in the low angle region $\left(2 \theta<10^{\circ}\right)$, which provides information on the confined d-spacing (basal spacing) between the silicate layers. In this case there are no well-defined WAXS basal reflections, only a broad reflection peak appearing at $2 \theta=4.7^{\circ}$ and corresponding to a $d_{001}$ basal spacing of $1.87 \mathrm{~nm}$. This broad maximum in PA6-NC is shifted to a lower $2 \theta$ value than the broad intense peak appearing at around $2 \theta=5^{\circ}$ for the pristine organoclay [28]. This shift indicates that the interlayer spacing of nanoclay stacks slightly increases from $1.8 \mathrm{~nm}$ to $1.87 \mathrm{~nm}$, suggesting that the PA6 molecules are penetrating into the silicate interlayer spacing. On the other hand, it is interesting to note that nanoclays are likely to be 
oriented in the injection moulding direction. This forced orientation is a result of the high shear rates during the injection moulding process.

\section{Figure 1}

Figure 2a shows a representative TEM micrograph for the electrospun nanofibres of pure PA6. Using formic acid as solvent and a combination of optimal electrospinning conditions ( $5 \mathrm{~cm}$ of working distance, $10 \mathrm{kV}$ of electrospinning voltage), the average diameter of electrospun fibres is approximately $50 \mathrm{~nm}$, with an extremely uniform size distribution (see Figure 2b,c) and without any sign of bead formation. The structure of electrospun nanofibres from PA6-NC is just identical to those from the pure PA6, except for the fact that they show a smaller difference in their size distribution. The typical internal morphology of the electrospun nanofibres containing $3.2 \mathrm{wt} \%$ nanoclays is shown in Eigure $2 \mathrm{~d}$. It is clearly seen that the nanoclays are much more extensively exfoliated and even more widely distributed within the electrospun fibres than in the injection moulded PA6-NC (see Figure 1). This can also be confirmed by the absence of the $d_{001}$ basal spacing in the WAXS patterns. The nanoclays are preferentially oriented parallel to the fibre axis. This is due to the higher draw ratio that imparts a larger stress on the fibre as it is being formed during the electrospinning process and gives rise to a proper alignment of the 2-dimensional nanoclays along the fibre axis. Finally, $t$ is interesting to note here that the electrospinnability is improved by the presence of nanoclays; in good agreement with the results from poly(MMA-co-MAA) / nanoclay nanocomposite systems recently reported by Wang et al. [35].

\section{Figure 2}

\subsection{Crystalline structure of the bulk materials}

Figure 3 shows WAXS diffractograms from the bulk PA6 and PA6-NC prior to electrospinning. For pure PA6 there are three strong reflections at $2 \theta=20.3^{\circ}, 21.3^{\circ}$ and $23.3^{\circ}$ : The diffraction peaks at $20.3^{\circ}$ and $23.3^{\circ}$ are associated to the $\alpha$-phase of PA6 crystallites, which arise from the $(200)$ and $(002)+(202)$ crystal planes (monoclinic unit cell: $a=0.956 \mathrm{~nm}, b=1.724 \mathrm{~nm}$ and $c=0.801 \mathrm{~nm}$ [36], with the $\mathrm{b}$ dimension being along the chain axis) [37]. The corresponding d-spacings are 0.44 $\mathrm{nm}$ and $0.37 \mathrm{~nm}$, respectively, which represent the projected intermolecular distance 
within the hydrogen bonded planes and the inter-planar distance. The diffraction peak at $2 \theta=21.3^{\circ}$ is characteristic of pseudo-hexagonal $\gamma$-phase crystals of PA6, which is associated to the (001) crystal planes (monoclinic structure with $a=0.933 \mathrm{~nm}$, $b=1.688 \mathrm{~nm}$ and $c=0.478 \mathrm{~nm}$ ) [38].

For PA6-NC with 3.2 wt $\%$ nanoclay, the predominant $\gamma$-form peak appears at $2 \theta=$ $21.3^{\circ}$ together with a small shoulder on the left side of this peak which represents some traces of $\alpha$-form PA6 crystals. Furthermore, an additional peak appears at $10.9^{\circ}$ upon loading nanoclays into the pure PA6. From the literature $[37,39,40]$, this latter peak is ascribed to the $\gamma$-crystalline structure, arising from its (020) plane. The above results indicate that, during processing of PA6-NC, nanoclays suppress the formation of $\alpha$-form crystals and significantly promote the formation of $\gamma$-crystals. Even though a small amount of $\gamma$-form crystals co-exists in the pure PA6, the $\alpha$-form population is the dominant crystalline phase for pure PA6. On the contrary, in PA6$\mathrm{NC}$, the $\gamma$ crystals are predominantly formed at expenses of the $\alpha$-crystals. This could be due to the fact that the nanoclays, which become exfoliated during melt processing, provide PA6 molecules with much more extensive contact surfaces to interact $[34,41]$. As a result, the mobility of the molecular chains is depressed and constrained to induce $\gamma$-form crystals rather than the $\alpha$-form ones.

\section{Figure 3}

\subsection{Crystalline structure of the electrospun nanofibres}

It is well established that uniaxial drawing, such as high-speed melt-spinning, determines the crystalline form of the resulting PA6 fibres, generally favouring the formation of the crystalline $\gamma$-phase rather than the $\alpha$-phase [42]. To study the effect of nanoclay on polymorphism in the electrospun nanofibres, WAXS measurements were conducted on both electrospun nanofibres of PA6 and PA6-NC. From Figure 4 it is obvious that both electrospun nanofibres exhibit just the two characteristic maxima at $2 \theta=10.4^{\circ}$ and $21^{\circ}$ which are readily confirmed as the $\gamma$-form crystals of PA6. The corresponding d-spacings are $0.756 \mathrm{~nm}$ and $0.417 \mathrm{~nm}$, which will be hereafter defined as $\gamma_{1}$ and $\gamma_{2}$, respectively. These WAXS data suggest that electrospinning behaves in the same manner as high-speed melt-spinning, predominantly giving rise to the $\gamma$-form crystalline structure that is most likely to be 
independent of the presence of the nanoclays. In other words, the presence of nanoclays does not significantly affect the crystalline structure formation of the PA6 matrix. These results are in good agreement with results from other authors, which confirm that the $\gamma$-form is preferred in both as spun and spun drawn fibres of pure PA 6 [42].

Fig. 4

As pointed out above, the electrospinning process is associated with a high shear stress and a very rapid structure formation of the polymer material. Accordingly, the ultra-high shear stress upon electrospinning not only initiates the alignment of nanoclays to a large extent (layer surface parallel to the fibre axis) but also induces an appreciable amount of macromolecules to change their crystal modification. The strong shear force results in a change of the spatial arrangement of the PA6 molecules, which could be envisaged as a twist of the hydrogen bonding between the oxygen in a carbonyl group and the hydrogen attached to a nitrogen between neighbouring polyamide molecular chains. This slight twist in the electrospun nanofibres produces a "shortening" of the chains which allows the formation of hydrogen bonds between parallel chains, thus constituting the crystalline $\gamma$-phase of PA6. In addition, the rapid solvent evaporation of the consolidating process of the electrospun nanofibres may also contribute to hinder the formation of perfect crystallites (such as those of the stable $\alpha$-phase). It is interesting to note that compared to the pure PA6 nanofibres, the electrospun nanofibres with nanoclays do not exhibit any well defined peaks of the crystalline $\alpha$-form at $2 \theta=20^{\circ}$ and $24^{\circ}$, but only some traces of them which could be considered as heterogeneities. Nevertheless, it is evident that the $\gamma$-form population is the dominant crystalline phase in all electrospun PA6-NC nanofibres.

\subsection{Temperature dependence of the crystalline structure in the electrospun nanofibres}

In order to study the thermal behaviour of the crystalline structure of the nanofibres WAXS measurements were carried out in a wide temperature range. Figure 5 shows the development of the WAXS patterns for the pure PA6 electrospun nanofibres as a function of temperature. During heating, the initial $\gamma_{1}$ peak $\left(2 \theta=10.8^{\circ}\right)$ is preserved 
up to $210^{\circ} \mathrm{C}$, and then abruptly disappears (Figure $5 \mathrm{a}$ ). On the contrary, the $\gamma_{2}$ peak $\left(2 \theta=21.3^{\circ}\right)$ shifts moderately with temperature to lower values, indicating that the $d$ spacing increases with temperature as a result of the thermal expansion of the $\gamma$ unit cell in its basal plane. At the final investigated temperature of $230^{\circ} \mathrm{C}$, a single broad peak appears at $2 \theta=19.0^{\circ}$ which is attributed to the so-called "amorphous" $\delta$-phase of PA6. The $\delta$-form developed above $230^{\circ} \mathrm{C}$ is believed to consist of very small crystallites with many defects caused by the breakage of hydrogen bonds.

On cooling (Figure $5 \mathrm{~b}$ ), below about $120^{\circ} \mathrm{C}$, the starting "amorphous" $\delta$-form is clearly splitted into two distinct peaks, $2 \theta=20.8^{\circ}$ and $22.4^{\circ}$, corresponding to d-spacings of $0.427 \mathrm{~nm}$ and $0.397 \mathrm{~nm}$, respectively. This double-peak nature in the WAXS pattern indicates that the sample crystallizes most likely in the $\alpha$-form. Because the peak positions are significantly different from the room temperature $\alpha$ peaks $\left(2 \theta=20.3^{\circ}\right.$ and $23.3^{\circ}$, with d-spacings $0.437 \mathrm{~nm}$ and $0.382 \mathrm{~nm}$, respectively), we hereafter define these peaks $\alpha_{1}{ }^{\prime}$ and $\alpha_{2}{ }^{\prime}$ as the meta-stable high-temperature crystalline $\alpha^{\prime}$ phase. Similar double diffraction peaks have already been reported by other authors $[43,44]$. On further decreasing the temperature, the $\alpha_{1}{ }^{\prime}$ peak shifts to a lower value, finally approaching $2 \theta=20.3^{\circ}$, while the $\alpha_{2}{ }^{\prime}$ peak changes towards a higher value of $2 \theta=23 \cdot 3^{\circ}$. These values are identical to those of the crystalline $\alpha$ form observed in bulk PA6 and PA6-NC prior to electrospinning, namely, the room temperature $\alpha$ phase. This means that upon cooling the distance between the H-bonded chains $\left(\alpha_{1}\right)$ increases, whereas the distance between chains bonded by van der Waals forces, i.e., hydrogen bonded sheets $\left(\alpha_{2}\right)$ decreases. Based on these results one can conclude that, on cooling from the melt to room temperature, an apparent crystal transition from $\alpha^{\prime}$-form to $\alpha$-form takes place in the electrospun pure PA6 nanofibres. Finally, after running an entire temperature cycle, i.e., heating and subsequent cooling, the initial $\gamma$-form crystals in the electrospun PA6 fibres completely turn into the $\alpha$-form crystals, with few remnants of $\gamma$-form crystals as deduced from the WAXS patterns. This behaviour is in good agreement with the general behaviour found for the crystallization from the melt of PA and on subsequent cooling [45].

\section{Figure 5}


On the other hand, the evolution of WAXS patterns for the electrospun PA6-NC nanofibres as a function of temperature is shown in Figure 6. While the heating behaviour is similar to that occurring in pure PA6 nanofibres, the evolution of WAXS patterns, on cooling and in the presence of nanoclays, is quite different. The noticeable feature observed here is that, from the melt to room temperature, the initial $\gamma$-form crystals are completely recovered without any intermediate formation of any $\alpha$ and/or $\alpha^{\prime}$-form of PA6, as can be observed in the pure PA6 nanofibres. In Table 1, the peak positions, the d-spacings and the polymorphic forms from all the studied systems are summarized.

Figure 6

Table 1

By plotting the variation of the WAXS d-spacings of the characteristic crystal forms as a function of temperature, a useful information on the polymorphism found in the electrospun nanofibres of PA6 and PA6-NC can be derived. Figure 7 shows the calculated Bragg d-spacings, from the two main reflections appearing during heating and cooling, for the pure PA6 and PA6-NC electrospun fibres. As mentioned above, the WAXS patterns obtained for the electrospun nanofibres, with and without nanoclay, clearly show that electrospinning primarily gives rise to the $\gamma$-form crystalline structure of PA6 and the corresponding d-spacings are almost independent of the presence of nanoclays (Figure 7a). In the temperature range from room temperature to $215^{\circ} \mathrm{C}$, the longer $\mathrm{d}$-spacing (open symbols) is practically constant. Above $\mathrm{T}=215^{\circ} \mathrm{C}$, it abruptly disappears. The constant d-spacing of the initial $\gamma_{1}(020)$ crystal planes is most likely an effect of the relative strong hydrogen bonding interactions. On the contrary, the evolution of the $\gamma_{2} 001 \mathrm{~d}$-spacings (solid symbols) upon heating is much more dependent on temperature than $\gamma_{1}$. This means that the $\gamma_{2}$ (001) planes are less thermally stable, i.e., they seem to be not so well crystallographically ordered as compared with the $\gamma_{1}(020)$ planes. It is worth pointing out here that the overall $d$-spacings for the electrospun nanofibres in the presence of nanoclays (squares) are slightly larger than those for the nanofibres without nanoclays (circles). 
Let us next follow the illustrative evolution of the initial $\gamma_{2}$-peak with temperature. Irrespective of the presence of nanoclays within the electrospun nanofibres the variation of the shorter $d$-spacing can be divided into four distinct temperature regimes represented as thick straight lines in Figure 7a: regime I (room temperature to $\left.90^{\circ} \mathrm{C}\right)$, regime II $\left(90^{\circ} \mathrm{C}-205^{\circ} \mathrm{C}\right)$, regime III $\left(205^{\circ} \mathrm{C}-220^{\circ} \mathrm{C}\right)$, and regime IV $\left(220^{\circ} \mathrm{C}\right.$ $230^{\circ} \mathrm{C}$ ).

- During the initial heating stage (regime I), molecular chains begin to be relaxed and, as a consequence, they become sufficiently mobile to provoke the thermal expansion of the $\gamma$ unit cell as indicated by the increase in $d$ spacings with temperature.

- In regime II, the d-spacings are nearly constant around a value of $0.427 \mathrm{~nm}$, which is surprisingly consistent with the $d$-spacing of the crystalline $\alpha_{1}{ }^{\prime}$-peak at $2 \theta=20.8^{\circ}$. This seems to indicate that, during the heating process, the crystal transition from $\gamma$ to $\alpha^{\prime}$ takes place at about $90^{\circ} \mathrm{C}$. In fact, the $\gamma-\alpha^{\prime}$ crystal transition in PA6 does not require substantial structural rearrangements, as only a cooperative twist of the molecular chains about the amide groups enables the $\mathrm{H}$-bonded parallel chains of the $\gamma$ form to switch to the $\mathrm{H}$-bonded anti-parallel chains of the $\alpha$ form [46-48]. In other words, this crystal transition $\gamma-\alpha$ is raised by a gradual solid-state reorganization rather than by a temperature-induced recrystallization process of highly defective crystals.

- The $\alpha^{\prime}$-form remains stable until $205^{\circ} \mathrm{C}$ and then in regime III, the $\mathrm{d}$-spacing gradually increases with temperature up to $220^{\circ} \mathrm{C}$. The final d-spacing at $220^{\circ} \mathrm{C}$ approaches a value of $0.382 \mathrm{~nm}$, which is attributed to the crystal $\alpha_{1}$ peak. This result evidently denotes another crystal transition $\alpha^{\prime}-\alpha$.

- Finally, in regime IV, the $d$-spacing for the transformed $\alpha_{1}$-crystal reflection drastically increases with increasing temperature, and finally merges into the "amorphous" $\delta$-phase.

\section{Figure 7}

After heating up to $230^{\circ} \mathrm{C}$, both electrospun nanofibres of PA6 and PA6-NC were cooled at a rather fast cooling rate of about $30 \mathrm{~K} / \mathrm{min}$. Figure $7 \mathrm{~b}$ displays the evolution of the WAXS d-spacings of both nanofibres during cooling from the melt 
down to room temperature. Depending on the presence of nanoclay or not, the development of the crystalline structure in both systems follows quite different routes. In the case of the pure PA6 electrospun fibres, at first, two distinct d-spacings (triangles) of $0.43 \mathrm{~nm}$ and $0.39 \mathrm{~nm}$ appear below $130^{\circ} \mathrm{C}$. This is the typical two-peak nature of the crystalline $\alpha$-form of PA6, which here corresponds to the high temperature reflections, $\alpha_{1}^{\prime}$ and $\alpha_{2}^{\prime}$, as mentioned above. The distance, $\Delta d=d\left(\alpha_{1}^{\prime}\right)-d\left(\alpha_{2}^{\prime}\right)=0.036 \mathrm{~nm}$, between these peaks is markedly smaller than $\Delta d=d\left(\alpha_{1}\right)-d\left(\alpha_{2}\right)=0.062 \mathrm{~nm}$ for the room temperature $\alpha$-form crystals. The increase in $\Delta d$ could be associated with an increase in crystalline density, crystallite size and crystalline perfection [49-52].

Continuing with the temperature decrease below $90^{\circ} \mathrm{C}$, the d-spacing for $\alpha_{1}$ remains nearly constant (solid triangles), whereas the $d$-spacing for $\alpha_{2}$ gradually shifts to much lower values (open triangles). Upon rapid cooling, the hydrogen bonds distributed within (200) planes of the a-modification presumably could not be perfectly rearranged. As suggested by Murthy et al., the higher the concentration of crystallographic mismatches within (200) sheets, the larger the departure of $\alpha_{1}$ and $\alpha_{2}$ spacings from the values of the ideal crystalline $\alpha$-form is observed [42]. Finally, once room temperature has been reached, the d-spacings approach the values of $0.44 \mathrm{~nm}$ and $0.37 \mathrm{~nm}$, similar to the typical reflections of the PA6 $\alpha$-form [53].

Briefly summarizing, the $d$-spacing of $\alpha_{2}$ is more sensitive to temperature than that of $\alpha_{1}$ because the latter, i.e., the distance between (200) planes, is mainly fixed by hydrogen bonds [54]. Therefore, it remains practically constant during cooling down below $80^{\circ} \mathrm{C}$. On the contrary, $d\left(\alpha_{2}\right)$, i.e., the inter-sheet distance, shows a dramatic decrease with lowering temperature. This suggests that a variation of the interplanar distance along the hydrogen bonds is more restricted than in the direction of the van der Waals interactions.

In contrast to the evolution of the $\alpha$-form crystallites in the electrospun pure PA6 nanofibres, for PA6-NC, the crystallization begins with the $\gamma$-crystalline form below about $150^{\circ} \mathrm{C}$ without evidence of any other crystal transition (squares). In addition, there are no significant changes on cooling in the d-spacings for both, $\gamma_{1}$ and $\gamma_{2}$. It is 
worth noting that the $\gamma$-form crystals always develop much earlier than the $\alpha$-form crystals. Furthermore, there are little changes in the pseudohexagonal crystals upon cooling. These results support the fact that the $\gamma$-form crystals are thermally more stable than the $\alpha$-form crystals. A most interesting finding, based on the variabletemperature WAXS measurements, is that while the $\gamma$-form crystals in the electrospun PA6 nanofibres completely turn into a-form upon heating and subsequent cooling, the evolution of the $\gamma$-form crystals in the electrospun PA6-NC nanofibres is totally reversible, which may be described as a "molecular memory effect"

Finally it is worth emphasizing that while the bulk PA6 exhibits, both, the fully extended all trans $\alpha$-form and the pleated sheeted $\gamma$-form crystalline structures, the melt processed PA6-NC with nanoclays predominantly develops $\gamma$-form crystals. In contrast, the electrospun fibres of both systems mainly consist of the $\gamma$-crystalline phase, whose development is fully independent of the presence of nanoclays. This can be explained as follows: The electrospinning process causes an extremely large effective draw ratio which aligns the anisotropic nanoclays as well as the polymer chains most likely parallel to the fibre direction. In addition, the rapid solidification process of stretched chains under high draw ratios during the last stages of electrospinning could hinder the most energetically stable $\alpha$-form due to lack of time for developing. As a consequence, the crystal form in electrospun nanofibres is preferentially locked in the $\gamma$-form for kinetical reasons.

\section{Conclusions and outlook}

The main results concerning the crystalline structures originated by electrospinning are the following:

1) Upon heating from room temperature to the melt, the crystalline $\gamma$-form firstly transforms into the $\alpha^{\prime}$-form, and then into the $\alpha$-form before melting proceeds; namely: $\gamma \rightarrow \alpha^{\prime} \rightarrow \alpha \rightarrow \delta$.

2) For pure PA6 electrospun nanofibre, when cooling from the melt to room temperature, first the "amorphous" $\delta$ phase develops and then the transformations $\delta \rightarrow \alpha^{\prime}$ and $\alpha^{\prime} \rightarrow \alpha$ take place. Both, the high temperature $\alpha^{\prime}$ 
and the room temperature a phases, are thermally unstable. The system of electrospun PA6-NC nanofibres also starts with the $\delta$ phase and then it undergoes a $\delta \rightarrow \gamma$ transition. The resulting $\gamma$-form crystals exhibit a relatively high thermal stability as compared with the $\alpha$-form crystals.

3) During cooling, the $\gamma$-form in the PA6-NC electrospun fibres seems to crystallize earlier than the $\alpha$-form in PA6.

4) For pure PA6 electrospun nanofibres, after a complete cycle of heating and subsequent cooling, the $\gamma$-form crystals are completely transformed into the $\alpha$ form crystals as in bulk PA6, whereas for the PA6-NC electrospun nanofibres the $\gamma$-form crystals are molecularly preserved.

The temperature dependent evolution of polymorphism in the electrospun nanofibres of pure PA6 and PA6-NC described above may give useful information for their use as reinforcing elements in polymer composites. It is known that the impact strength of PA6 containing mostly $\gamma$-form crystals is markedly higher than when it contains $\alpha$ form crystals. During processing (e.g., hot compaction) of electrospun nanofibres, which incorporate as reinforcing elements either PA6 or PA6-NC, the $\gamma$-form crystals can be readily transformed into $\alpha$-form ones in the first case; but they remain unchanged for the electrospun nanofibres of PA6-NC. As a consequence, the toughness of nanocomposites reinforced with electrospun nanofibres of PA6-NC should be quite stable with temperature. This has been recently confirmed by some of us and the results will be published soon.

\section{Acknowledgements}

The authors thank Dr. Fornes and Prof. Paul from the Department of Chemical Engineering and Texas Materials Institute, University of Texas at Austin, for kindly supplying the PA6-clay nanocomposites. F.A. and F.J.B.C wish to acknowledge the "Ministerio de Educación y Ciencia" (grant FIS2004-01331), Spain, for the support of this investigation. The European Community - Research Infrastructure Action under the FP6 "Structuring the European Research Area" Programme, IA-SFS Contract RII3-CT-2004-506008, funded the WAXS measurements at HASYLAB (DESY). 
Technical assistance from Dr. S. S. Funari, Max Planck Institute for Colloids and Surfaces (Hamburg), is specially acknowledged. 


\section{References}

[1] Shonaike GO, Advani SG. Advanced Polymeric Materials: Structure Property Relationships. C.R.C. Press, Boca Raton, FL, USA, 2003.

[2] Giannelis EP. Adv. Mater. 1996;8:29.

[3] Zilg C, Reichert P, Dietsch F, Engelhard T, Mülhaupt R. Kunststoffe 1998;88:10.

[4] Pinniavaia TJ, Beall G. editors. Polymer-Clay Nanocomposites. New York: Wiley, 2001.

[5] Komarneni S. J Mater Chem 1992;2:1219.

[6] Rocco MC, William RS, Aliviaiatos P. editors. Nanotechnology Research Directions: IWGN Workshop Report, National Science and Technology Council, September 1999

[7] Kim GM, Lee DH, Hoffmann B, Kressler J, Stöppelmann G. Polymer 2001;42:1095.

[8] Creasy TS, Kang YS. J. Thermoplast. Compos. 2004;17:205.

[9] Karger-Kocsis J. Polym Bull 1996;36:119

[10] Hine P, Ward I. Materials World 1998;6:608.

[11] Kim GM, Qin H, Fang X, Sun FC, Mather PT. J Polym Sci B: Polym Phys 2003;41:3299.

[12] Thostenson ET, Zhifeng R, Chou-Tsu W. Compos. Sci. Technol. 2001;61:1899.

[13] Andrews R, Jacques D, Minot M, Rantell T. Macromol. Mater. Eng. 2002;287:395.

[14] Gojny FH, Nastalczyk J, Roslaniec Z, Schulte K. Chem. Phys. Lett. 2003;370:820.

[15] Kim GM, Wutzler A, Radusch HJ, Michler GH, Simon P, Sperling RA, Parak WJ. Chem. Mater. 2005;17:4949.

[16] Li D, Xia Y. Adv. Mater. 2004;16:1151.

[17] Shin YM, Hohman MM, Brenner MP, Rutledge GC. Polymer 2001;42:9955.

[18] Deitzel JM, Kleinmeyer J, Harris D, Beck TNC. Polymer 2001;42:261.

[19] Bognitzki M, Hou H, Ishaque M, Frese T, Hellwig M, Schwarte C, Schaper A, Wendorff JH, Greiner A. Adv. Mater. 2000;12:637.

[20] Reneker DH, Chun I. Nanotechnology 1996;7:216.

[21] Li L, Bellan LM, Craighead HG, Frey MW. Polymer 2006;47:6208.

[22] Kim GM, Lach R, Michler GH, Chang CH. Macromol. Rapid Commun. 2005;26:728.

[23] Fong H, Liu W, Wang CS, Vaia RA. Polymer 2002;43:775.

[24] Dror Y, Salalha W, Khalfin RL, Cohen Y, Yarin AL, Zussman E. Langmuir 2003;19:7012.

[25] Salalha W, Dror Y, Khalfin RL, Cohen Y, Yarin AL, Zussman E. Langmuir 2004;20:9852. 
[26] Kim GM, Michler GH, Pötschke P. Polymer 2005;46:7346.

[27] Wang M, Singh H, Hatton TA, Rutledge GC. Polymer 2004;45:5505.

[28] Griffith AA. Phil. Trans. R. Soc. Ser. A. 1920;221:163.

[29] Baltá-Calleja FJ, Fakirov S. Microhardness of Polymers. Cambridge: Cambridge University Press, 2000.

[30] Heuvel HM, Huisman R. J. Appl. Polym. Sci. 1981;26:713.

[31] Stephen JS, Chase DR, Rabolt JF. Macromolecules 2004;37:877.

[32] Ibanes C, de Boissieu M, David L, Seguela R. Polymer 2006;47:5071

[33] Fornes TD, Yoon PJ, Keskkula H, Paul DR. Polymer 2001;42;9929.

[34] Fornes TD, Paul DR. Polymer 2003;44:3945.

[35] Wang M, Hsieh AJ, Rutledge GC. Polymer 2005;46:3407.

[36] Arimoto H. J. Polym. Sci. Part A 1964;2:2283.

[37] Wunderlich B. Macromolecular Physics, Vol. 1. Academic Press: New York, 1973.

[38] Xenopoulos A, Clark ES. In: Kohan MI, editor. Nylon plastics handbook, New York: Hanser, 1995.

[39] Yuan M, Turng LS. Polymer 2005;46:7273.

[40] Lincoln DM, Vaia RA, Wang ZG, Hsiao BS. Polymer 2001;42:1621.

[41] Wu Q, Liu X, Berglund LA. Polymer 2002;43:2445.

[42] Murthy NS, Aharoni SM, Szollosi AB. J. Polym. Sci. B 1985;23:, 2549.

[43] Li L, Koch MHJ, de Jeu WH. Macromolecules 2003;36:1626.

[44] Ramesh C. Macromolecules 1999;32: 5704.

[45] Ramesh C. Macromolecules 1999;32:3721.

[46] Miyasaka K, Makishima K. J. Polym. Sci. Part A1 1967;5:3017.

[47] Penel-Pierron L, Seguela R, Lefebvre JM, Miri V, Depecker C, Jutigny M, Pabiot J. J. Polym. Sci. Polym. Phys. 2001;39:1224.

[48] Aharoni SM. n-Nylons: their synthesis, structure and properties. Wiley: New York, 1997.

[49] Ibanes C, Boissieu MD, David L, Sequela R. Polymer 2006;47:5071.

[50] Holmes DR, Bunn CW, Smith DJ. J. Polym. Sci. 1955;17:159.

[51] Murthy NS, Curran SA, Aharoni SM, Minor H. Macromolecules 1991;24:3215.

[52] Ramesh C, Gowd EB. Macromolecules 2001;34:3308.

[53] Wunderlich B, Grebowicz J. Adv. Polym. Sci. 1984;60-61:1.

[54] Bunn CW, Garner EV. Proc. R. Soc. A 1947;189:39.

[55] Heuvel HM, Huisman R, Lind KCJB. J. Polym. Sci. Phys. 1976;14:921. 


\section{Captions for Figures \& Table}

Figure 1: TEM micrograph showing the dispersion of nanoclays in injection molded PA6-NC. The insert illustrates the WAXS pattern of the bulk material and the position of the broad reflection peak (arrow).

Figure 2: a) TEM micrograph of electrospun nanofibres of pure PA6; b) and c) fibre diameter distribution for PA6 and PA6-NC fibres respectively; d) TEM detail of the internal morphology of electrospun nanofibres containing $3.2 \mathrm{wt} \%$ nanoclay.

Figure 3: WAXS patterns of bulk PA6 and PA6-NC.

Figure 4: WAXS patterns of electrospun PA6 and PA6-NC fibres.

Figure 5: Variation of the WAXS patterns for PA6 electrospun nanofibres: a) with increasing temperature and b) upon cooling from the melt. The scans are shifted
along the $y$-axis for clarity.

Figure 6: WAXS variation of electrospun fibres from PA6-NC: a) with increasing temperature and $b$ ) during cooling from the melt. The scans are shifted along the $y$ -

Figure 7: Changes in d-spacings during heating (a) and cooling (b) for PA6 and PA6$\mathrm{NC}$ electrospun nanofibres. The lines drawn through the points are a guide for the eye and the arrows indicate the corresponding $y$-axes.

Table 1. Peak positions observed in all systems, corresponding d-spacings and crystal form assignment. 